\title{
An Ultra-Violet Tolerant Wild-Type Strain of Melanin-Producing Bacillus thuringiensis
}

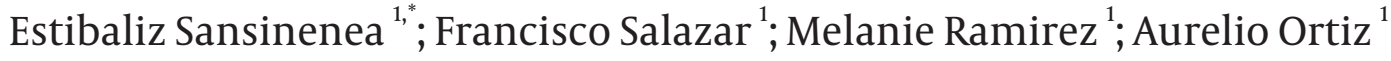 \\ ${ }^{1}$ Faculty of Chemical Sciences, Meritorious Autonomous University of Puebla, Mexico \\ ${ }^{*}$ Corresponding author: Estibaliz Sansinenea, Faculty of Chemical Sciences, Meritorious Autonomous University of Puebla, Mexico. Tel: +52-2222295500, Fax: +52-222229558, \\ E-mail: estisan@yahoo.com
}

Received: June 2, 2014; Revised: September 5, 2014; Accepted: October 10, 2014

\begin{abstract}
Background: Bacillus thuringiensis is the most successful biological control agent used in agriculture, forestry and mosquito control. However, the insecticidal activity of the $B$. thuringiensis formulation is not very stable and rapidly loses its biological activity under field conditions, due to the ultraviolet radiation in sunlight. Melanin is known to absorb radiation therefore photo protection of $B$. thuringiensis based on melanin has been extensively studied.

Objectives: The aim of this study was to find a wild type strain of naturally melanin-producing B. thuringiensis to avoid any mutation or manipulation that can affect the Cry protein content.

Materials and Methods: Bacillus thuringiensis strains were isolated from soils of different States of Mexico and pigment extraction was followed by lowering the $\mathrm{pH}$ to 2 using $1 \mathrm{~N} \mathrm{HCl}$. Pigment was characterized by some chemical tests based on its solubility, bleaching by $\mathrm{H}_{2} \mathrm{O}_{2}$ and flocculation with $\mathrm{FeCl}_{3}$, and using an Infrared (IR) spectrum. Ultraviolet (UV) irradiation experiment was performed to probe the melanin efficacy.

Results: ELI52 strain of B. thuringiensis was confirmed to naturally produce melanin. The Cry protein analysis suggested that ELI52 is probably a B. thuringiensis subsp. israelensis strain with toxic activity against the Diptera order of insects. Ultra Violet protection efficacy of melanin was probed counting total viable colonies after UV radiation and comparing the results with the non-producing melanin strain L-DOPA (L-3, 4-dihydroxyphenylalanine) was also detected in the culture. ELI52 strain showed an antagonistic effect over some common bacteria from the environment.

Conclusions: ELI52 wild-type strain of B. thuringiensis is a good bio-insecticide that produces melanin with UV-resistance that is probably toxic against the Diptera order of insects and can inhibit the growth of other environmental bacteria.
\end{abstract}

Keywords: Biological Control Agents; Melanin; Ultraviolet Rays; Bacillus thuringiensis

\section{Background}

Bacillus thuringiensis is a Gram-positive, spore-forming, soil bacteria and the most successful biological control agent that produces distinctly shaped crystals during sporulation. These crystals are composed of proteins known as insecticidal crystal proteins (Cry), which are selectively toxic to different species of several invertebrate phyla. Therefore, B. thuringiensis has been used as a bio pesticide in agriculture, forestry and mosquito control and today $B$. thuringiensis is the most widely used bio-pesticide in the world (1). However, one of the main disadvantages is that the insecticidal activity of $B$. thuringiensis formulation is not very stable and rapidly loses its biological activity under field conditions, due to the Ultraviolet (UV) radiation of sunlight (2). Therefore, the use of $B$. thuringiensis as a bio-insecticide is more expensive than other alternatives because repeated spraying is necessary.

Melanin is known to absorb radiation consequently photo protection of $B$. thuringiensis based on melanin has been studied extensively. Liu et al. (2) reported the use of melanin pigment extracted from Streptomyces lividans for photo protection of larvicidal activity of a mosquitocidal strain of B. thuringiensis subsp. israelensis. Patel et al. (3) and Saxena et al. (4) obtained a melanin-producing mutant by artificial mutation of $B$. thuringiensis subsp. kursta$k i$ after successive rounds of UV radiation. Although compared with their parent strains the two mutants had a higher UV resistance, this mutation had some negative impacts on them, such as the loss of some Cry genes.

Several research groups have obtained B. thuringiensis mutants producing melanin after treatment with a mutagenic agent (5-7). Although, these mutants were more resistant to UV radiation, some of them lost their toxinencoding genes.

Ruan et al. (8) claimed that $B$. thuringiensis strains have the potential to produce melanin in the presence of $\mathrm{L}$ tyrosine at an elevated temperature $\left(42^{\circ} \mathrm{C}\right)$. However, at this temperature, insecticidal Cry proteins could not be

Copyright (C) 2015, Ahvaz Jundishapur University of Medical Sciences. This is an open-access article distributed under the terms of the Creative Commons Attribution-NonCommercial 4.0 International License (http://creativecommons.org/licenses/by-nc/4.0/) which permits copy and redistribute the material just in noncommercial usages, provided the original work is properly cited. 
synthetized, thus genetic engineering is needed to produce insecticidal proteins in B. thuringiensis mutants producing melanin (9). In this study, we report on a wild type strain of B. thuringiensis capable of naturally producing melanin and Cry proteins with antagonistic effects over some common bacteria of the environment such as Micrococcus luteus, Staphylococcus aureus, Klebsiella pneumoniae and Serratia marcescens. The characteristics and UV protection efficacy of melanin in B. thuringiensis formulations after UV radiation are also described. L-DOPA was also detected in the culture. The protein pattern showed that this $B$. thuringiensis strain probably belongs to subsp. israelensis.

\section{Objectives}

Several research groups have obtained B. thuringiensis mutants producing melanin by successive rounds of UV radiation or by treatment with mutagenic agents, however these mutants lose some of their toxin-encoding genes. In the present study we aimed to find a wild type strain of naturally melanin-producing B. thuringiensis, with resistance to UV radiation to avoid any mutation or manipulation that can affect the Cry protein contents.

\section{Materials and Methods}

\subsection{Bacterial Strains and Growth Conditions}

Bacillus thuringiensis ELI2 strain was isolated from soil of the North of Mexico and ELI52 strain was isolated from soil of South of Mexico, as a brown pigment producer. Samples of soil were collected from uncultivated soil, which did not include organic matter or inorganic waste. Both strains were grown at $30^{\circ} \mathrm{C}$ with shaking at $200 \mathrm{rpm}$, in Luria Bertani (LB), Brain Heart Infusion (BHI), Nutrient Broth (NB), Tris G (13) and Trypticase Soy Broth (TSB) medium, to determine which medium was the best for the production of melanin. M. luteus, S. aureus, K. pneumoniae and $S$. marcescens strains were grown at $30^{\circ} \mathrm{C}$ with constant shaking in LB medium.

\subsection{Pigment Characterization}

Cultures of $B$. thuringiensis ELI52 were grown in LB medium at $200 \mathrm{rpm}$ and $30^{\circ} \mathrm{C}$ for 20 hours and then centrifuged at $6000 \mathrm{rpm}$ for 20 minutes. The cell mass was removed and the pigment in the supernatant was precipitated by lowering the $\mathrm{pH}$ to 2 using $1 \mathrm{~N} \mathrm{HCl}$. The precipitate was collected by centrifugation and was dried at room temperature. The dried pellet was mixed with potassium bromide and then IR spectrum was measured on a Magna-IR Spectrometer 750 Nicolet FT-IR spectrophotometer. Synthetic melanin from Sigma Aldrich (USA) was used as a standard. Melanin was also identified on the basis of the following characteristics: solubility in $1 \mathrm{M}$ $\mathrm{KOH}$, hot $0.5 \mathrm{M} \mathrm{NaOH}$ and $1 \mathrm{M} \mathrm{Na}_{2} \mathrm{CO}_{3}$ and insolubility in organic solvents (such as acetone, chloroform, methanol, ethanol, ether and water), bleaching by $\mathrm{H}_{2} \mathrm{O}_{2}$ (15\%), and formation of a flocculent brown precipitate upon addition of $0.1 \mathrm{M} \mathrm{FeCl}_{3}$ (4).

\subsection{Production of L-DOPA}

The culture medium was centrifuged at $6000 \mathrm{rpm}$ for 15 minutes, followed by filtration through $0.45 \mu \mathrm{m}$ poresize membrane filter. The filtrate was tested for the production of L-3, 4-dihydroxypohenylalanine (L-DOPA) using the chemical method of Arnow (10).

\subsection{Biomass Generation}

Cultures of B. thuringiensis ELI 2 and ELI52 were grown in $100 \mathrm{~mL}$ of $\mathrm{BHI}$ at $200 \mathrm{rpm}$ and $30^{\circ} \mathrm{C}$ during 72 hours. After this time period, when spores and crystals were released by the bacteria in the medium as judged by phase contrast microscopy, the supernatants were heated at $65^{\circ} \mathrm{C}$ for 40 minutes to kill viable vegetative cells. Next, biomass was collected using the lactose-acetone coprecipitation method (11).

\subsection{Ultra Violet Irradiation}

Biomass was suspended in $0.05 \mathrm{M}$ potassium phosphate buffer at pH 6.8. Samples $(10 \mathrm{~mL})$ of these suspensions were irradiated (with either $254 \mathrm{~nm}$ or $365 \mathrm{~nm}$ ) in petri plates at a distance of $30 \mathrm{~cm}$ from the UV source for several discrete episodes. Aliquots $(100 \mu \mathrm{L})$ were removed from the irradiated samples at different time intervals, plated on LB agar plates and incubated at $30^{\circ} \mathrm{C}$ to determine total viable count. The total viable count was determined by counting the colonies that grew on the next day after UV radiation and comparing with the colonies that grew without UV irradiation. The experiment was also performed with cells of ELI2 and ELI52 strains. The cells of both strains were suspended in $0.85 \%$ sterilized $\mathrm{NaCl}$ solution, mixed and irradiated at the same distance and at the same time as the biomass. The experiment was carried out three times.

\subsection{Protein Analysis}

After growing $B$. thuringiensis for 72 hours, until the release of spores and crystal proteins, the cells were recovered by centrifugation and resuspended in Tris-Ethylenediaminetetraacetic acid (Tris-EDTA) buffer at pH 7.5 and 0.1 M. Next, a small amount of lysozyme was added and the sample was incubated for 30 minutes at $37^{\circ} \mathrm{C}$. These protein samples were then completely solubilized by incubation for five minutes at $100^{\circ} \mathrm{C}$ in Sodium Dodecyl Sulfate (SDS)/sample buffer (50 mM-Tris- $\mathrm{HCl}$ (pH 6.8), 1\% SDS (w/v), 25 mM- $\beta$-mercaptoethanol, 2 mM phenylmethanesulfonyl fluoride, $10 \%$ (w/v) glycerol and $0.0025 \%(w / v)$ bromophenol blue). Proteins were fractioned and observed on $8 \%$ Sodium Dodecyl Sulfate in Polyacrylamide Gel Electrophoresis (SDS-PAGE) (12). The molecular weight SDS-PAGE standard was from Bio-rad (USA). 


\subsection{Antagonistic Activity Against Environment Bacteria}

Cultures of M. luteus, S. aureus, K. pneumoniae and S. marcescens bacteria were prepared by suspension in $3 \mathrm{~mL}$ of LB and incubated at $30^{\circ} \mathrm{C}$ with constant shaking at $200 \mathrm{rpm}$ for 20 hours to reach a cell density of approximately $1 \times 10^{8}$ colony forming units (CFU) per milliliter. With the cultures in suspension, dilutions were prepared to seed the Petri dishes for the paired bacterial confrontation trials. The dilutions were prepared for all microorganisms to reach $1 \times 10^{5}$ colony forming units (CFU) per milliliter. Bacillus thuringiensis strains were seeded with a bacteriological loop forming a rectangle (Figure 1) in LB agar medium. The other environment bacteria suspensions were seeded in a straight line crossing the rectangle diagonally to create a population gradient in the simple streak. The dilutions were used to create "crossed rectangle" tests with three proportions of $B$. thuringiensis to other environment bacterial strains, including 1:10, 1:1 and 10:1, respectively (13). The inoculated Petri dishes were incubated at $30^{\circ} \mathrm{C}$ for 24 hours and the types of different bacteria growth inhibition was recorded.

\section{Results}

\subsection{Pigment Production}

The number of colonies isolated from different soil samples was variable depending of the nature of the soil. On average, in these soil samples the number of Bacillus sp. colonies was between four and ten per $100 \mu \mathrm{L}$ of soil suspension that was plated on the plates. Bacillus thuringiensis ELI52 strain produced pigment in a different media with no L-tyrosine, giving colonies, which were dark brown in color, 21 hours after inoculation. However, the intensity of the color of the pigment was highest in the BHI medium (Table 1). Therefore, this medium was selected for the next experiments. The maximum production was in the late exponential phase of growth after 21 hours of inoculation.

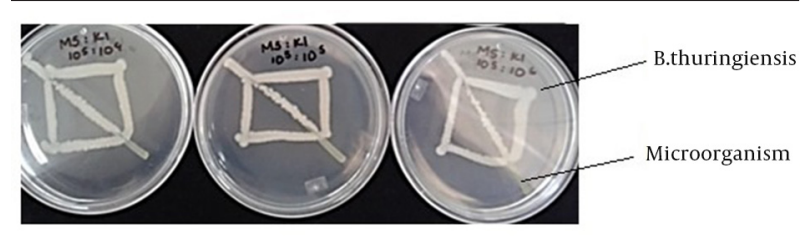

Figure 1. Streaking in the Crossed Rectangle Used for the Qualitative Evaluation of Bacillus Thuringiensis Interaction With Other Environment Bacteria

Table 1. Intensity of the Pigment Produced by Bacillus thuringiensis ELI52 Grown on Different Agar Media for 21 Hours at $30^{\circ} \mathrm{C}$

\begin{tabular}{lc}
\hline Media & Pigment Intensity \\
\hline Nutrient Agar (NA) & + \\
Tris G salts agar & + \\
Luria Bertani (LB) agar & ++ \\
Trypticase Soy Agar (TSA) & +++ \\
Brain Heart Infusion agar (BHI) & ++++ \\
\hline
\end{tabular}

\subsection{Pigment Characterization}

Primary characterization indicated that the brown recovered pigment was melanin. The pigment was insoluble in organic solvents (such as methanol, butanol, isopropanol, ether and acetone) and soluble in alkaline conditions such as $1 \mathrm{M} \mathrm{KOH}$ and $1 \mathrm{M} \mathrm{Na}_{2} \mathrm{CO}_{3}$. The dark brown pigment was bleached to a colorless state by addition of $\mathrm{H}_{2} \mathrm{O}_{2}(15 \%)$ and a brown precipitate was formed when $0.1 \mathrm{M} \mathrm{FeCl}_{3}$ was added to the pigment solution. The Fourier Transform Infrared (FTIR) spectra of the pigment extracted from $B$. thuringiensis and the standard melanin were compared and showed absorbance peaks around $3300 \mathrm{~cm}^{-1}$ and $1650 \mathrm{~cm}^{-1}$, due to the absorbance of $-\mathrm{OH},-\mathrm{NH}_{2}$ and $\mathrm{C}=\mathrm{O}$ respectively (Figure 2). The culture broth of the melanin-producing ELI52 strain was tested qualitatively for the presence of L-DOPA. When tested by the method of Arnow (10) there was formation of a red-orange color, indicating the presence of L-DOPA, which appeared in the culture of ELI52 during the lateexponential phase of growth, between the 20th and 21st of growth. The concentration of L-DOPA was not measured quantitatively.

\subsection{Ultra Violet Resistance}

The comparative survival of the melanin-producing $B$. thuringiensis strain ELI52 and melanin non-producing strain ELI2 by UV irradiation at $254 \mathrm{~nm}$ and $365 \mathrm{~nm}$, are shown in Figures 3 and 4, respectively. The experiment was done with biomass and cells at the same time and was repeated three times. At both wavelengths, the viability of the ELI2 was inactivated much faster than of the melanin-producing strain ELI52. However, this phenomenon was more significant at $254 \mathrm{~nm}$ than $365 \mathrm{~nm}$. This effect was shown both with cells and biomass, yet the biomass was more relevant. Therefore, it can be concluded, that melanin produced by ELI52 improved the resistance of the spores and vegetative cells to UV irradiation.

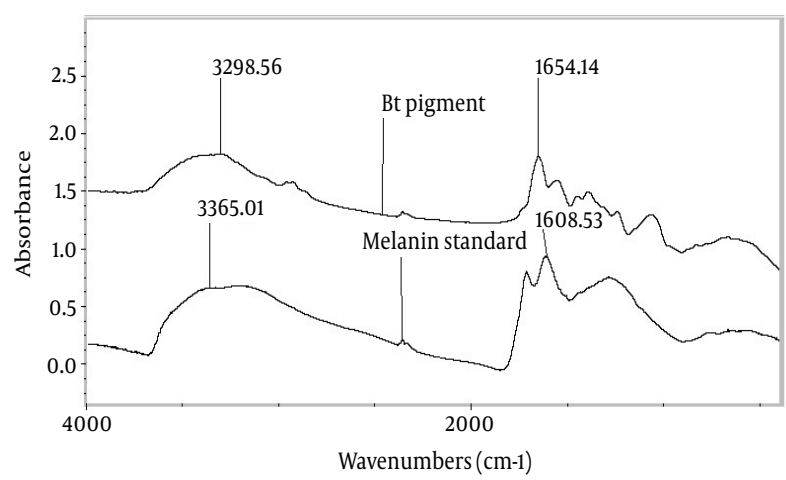

Figure 2. The Fourier Transform Infrared Spectra of the Pigment Produced by Bacillus thuringiensis ELI52 and Standard Melanin 

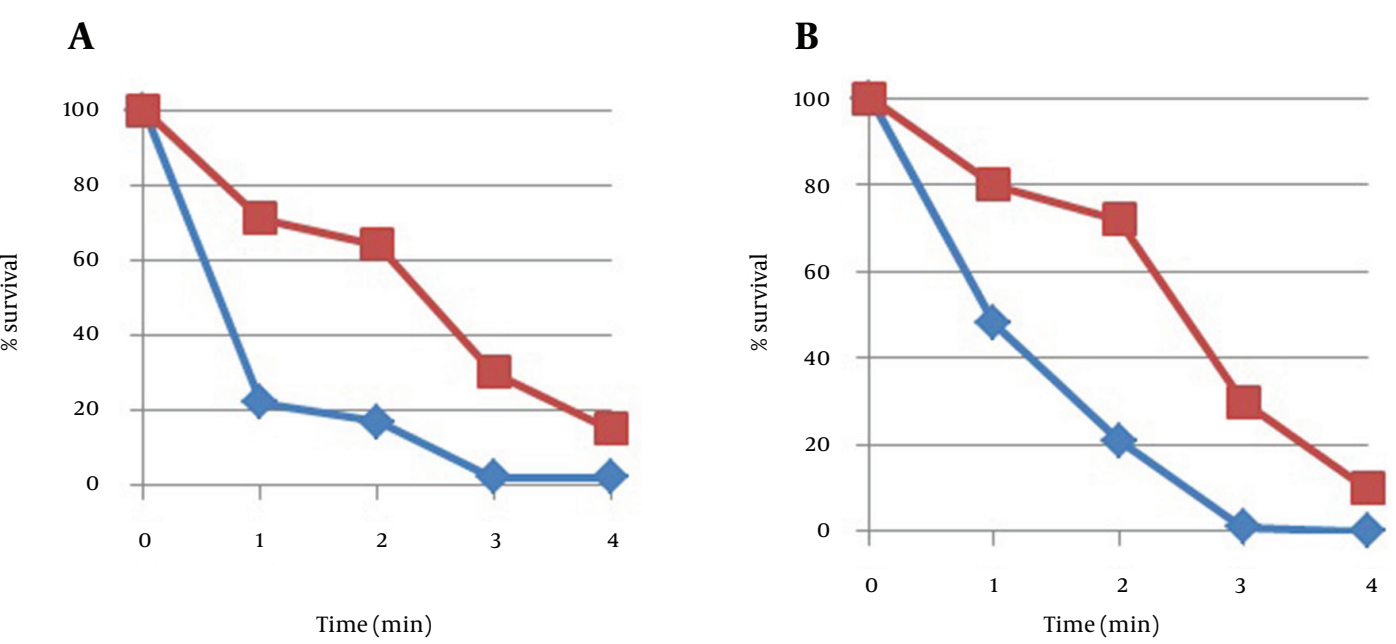

Figure 3. Effect of Ultra Violet Irradiation at $254 \mathrm{~nm}$ on Biomass A) and Cells B) of ELI2 (•) and ELI52 (•)
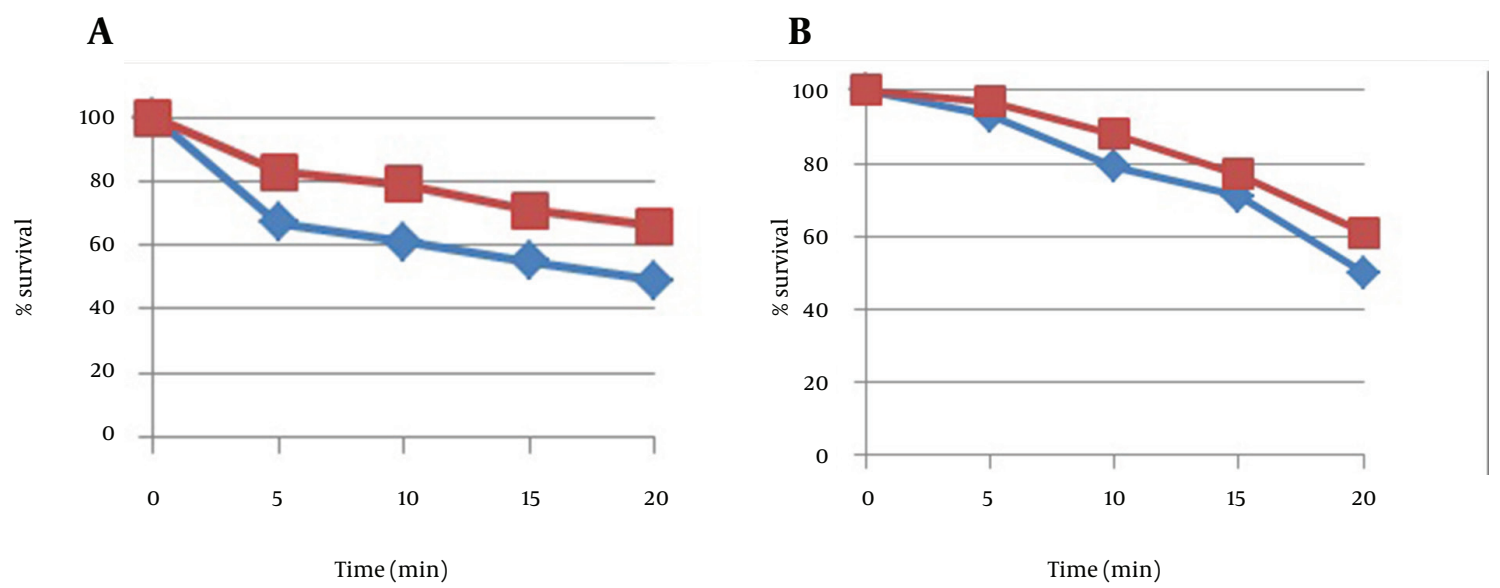

Figure 4. Effect of Ultra Violet Irradiation at $365 \mathrm{~nm}$ on the Biomass A) and Cells B) of ELI2 ( $)$ and ELI52 (घ)

\subsection{Insecticidal Crystal Protein Analysis in Bacillus thuringiensis Strains ELI52 and ELI2}

Melanin producer strains, ELI52 and ELI2, were incubated until the sporulation phase, when the crystal protein analysis was performed using SDS-PAGE gels. As shown in Figure 5, the major bands of the ELI52 strain included $130-\mathrm{KDa}$ protein corresponding to the Cry4A toxin, 68-KDa protein corresponding to the Cry11A toxin and $28-\mathrm{KDa}$ protein corresponding to the Cyt1A toxin (lanes 2 and 5). These data of the protein analysis along with the crystal morphology (by phase contrast microscopy observation), presenting a spherical form, indicated that ELI52 strain was probably $B$. thuringiensis subsp. Israelensis, which is toxic to larvae of a wide range of mosquito species. The ELI2 strain presented other bands quite different to that of the ELI52 strain (lanes 1 and 6). However, more studies are needed to identify this strain at the subspecies level.

\subsection{Analysis of the Confrontation of Bacillus} thuringiensis Against Ambient Bacteria

The culture conditions described in this study favored the growth of $B$. thuringiensis, and its interactions with other environment bacteria in a paired challenge model could be either beneficial or antagonistic, depending on the abundance of growth in LB. For the qualitative confrontation test, a crossed rectangle experiment (Figure 1) was designed. The types of growth of the different bacteria that was observed in the crossed rectangle model ranged from inhibition of bacteria in the first zone of interaction with $B$. thuringiensis to absence of inhibition along the entire streak, as shown in Table 2. As an approximation of the different environments in which microbial populations can vary, different proportions of bacteria were also used in the confrontation trials. In this way, it was observed that antagonism is more marked when there is a population of B. thuringiensis that is bigger than the population of the other bacteria. 
Sansinenea $E$ et al.

\begin{tabular}{lccccc}
\hline \multicolumn{1}{l}{ Table 2. Effect of Co-Culture of Bacillusthuringiensis With Other Microorganisms, Based on the Crossed-Rectangle Model Experiment } \\
\hline B. thuringiensis Strain & Dilution $^{\text {a }}$ & S. marcescens & M. luteus & K.pneumoniae & S. aureus \\
\hline ELI2 & $1: 10$ & 1 & 2 & 3 & 2 \\
& $1: 1$ & 1 & 2 & 3 & 1 \\
& $10: 1$ & 1 & 1 & 3 & 2 \\
ELI52 & $1: 10$ & 1 & 1 & 2 & 1 \\
& $1: 1$ & 1 & 1 & 1 & 1 \\
\hline
\end{tabular}

a The relation of dilutions indicate $B$. thuringiensis versus other microorganism. 1) Inhibition in the first streak, 2) inhibition in the second streak,3) no inhibition of growth of the environment strain versus B. thuringiensis.

Figure 5. Protein Analysis by Sodium Dodecyl Sulfate in Polyacrylamide Gel Electrophoresis of Bacillus thuringiensis Strains ELI52 and ELI2 Expressing the Cry Genes

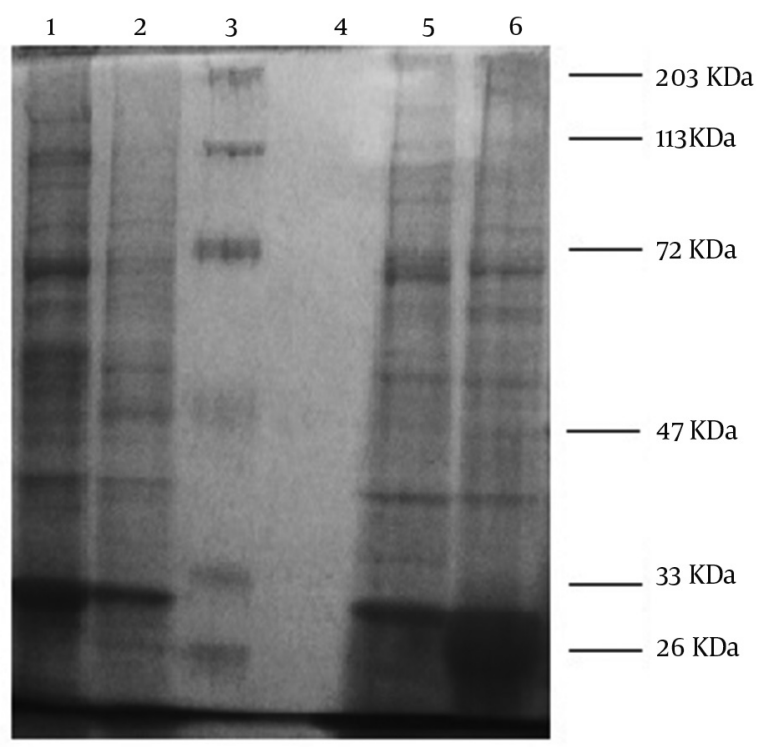

Lanes: 1 and 6, B. thuringiensis ELI2 $10 \mu \mathrm{L}$ and $15 \mu \mathrm{L}$, respectively; 2 and 5, B. thuringiensis ELI52 $10 \mu \mathrm{L}$ and $15 \mu \mathrm{L}$ respectively; 3, molecular weight marker; 4, blank.

\section{Discussion}

The use and acceptance of entomopathogen bacteria such as B. thuringiensis depends on prolonging the survival of the bacteria under field conditions to avoid repeated spraying. Sunlight irradiation is critical in the loss of biological activity of $B$. thuringiensis preparations due to UV irradiation at wavelengths ranging from 250 to $380 \mathrm{~nm}$, which damages the spores as well as their $\delta$-endotoxins $(2,14,15)$. To overcome the problem of sensitivity to UV irradiation of $B$. thuringiensis, some chemical screens have been found $(16,17)$. However, these chemical screens have some negative impacts on the environment. Melanin is a natural pigment that is easily biodegradable in the nature and can absorb radiation therefore it is a perfect photo protective agent.
Several research groups have obtained B. thuringiensis mutants producing melanin by successive rounds of UV radiation $(3,4)$ or by after treatment with a mutagenic agents (5-7). Although, these mutants were more resistant to UV radiation, some of them lost their toxin-encoding genes, due to mutations affecting the cry genes.

In this work, B. thuringiensis ELI52 was isolated as a wild strain with UV resistance and it was proven to produce melanin, therefore the insecticidal protein content was not affected due to any mutation or manipulation. The FT-IR spectrum of melanin produced by ELI52, was similar to that of the standard melanin from Sigma. Also the presence of L-DOPA was positively checked. This strain does not need L-tyrosine for the production of melanin. Therefore, the production of pigment by this strain is constitutive, thus this strain did not lose its insecticidal proteins and could tolerate UV with its insecticidal capacity unaffected.

Protein analysis of the crystals produced by ELI52 strain showed that this strain was probably B. thuringiensis subsp. israelensis, however, more studies are needed to identify the strain at the subspecies level. The antagonistic effect exerted by $B$. thuringiensis ELI52 could be due to secondary metabolites produced to act against different bacteria from the environment (18). This strain is a good bio-insecticide with UV-resistance that is probably toxic against the Diptera order of insects and can inhibit the growth of other environment bacteria. Therefore, it can be an economically cheaper option because repeated spraying is unnecessary. In this sense, we are working to determine the antimicrobial activity of the extract of this strain against several bacteria.

\section{Acknowledgements}

We wish to thank the staff of the laboratory of organic chemistry of the faculty of chemical sciences of the Meritorious Autonomous University of Puebla for their support.

\section{Authors' Contributions}

Here we confirm that Estibaliz Sansinenea and Aurelio Ortiz developed the original idea and the protocol, abstracted and analyzed the data, and wrote and revised 
the manuscript. Francisco Salazar and Melanie Ramirez carried out the experimental procedures.

\section{Funding/Support}

This study was performed with a grant from VIEP (Vicerrectoría de Investigación y Estudios de Posgrado) (project SARE-NAT13 - 1) and PROMEP (Programa para el Mejoramiento del Profesorado) (project PTC-333).

\section{References}

1. Sansinenea, E. Netherlands: Springer; 2012. Bacillus thuringiensis biotechnology.

2. Liu YT, Sui MJ, Ji DD, Wu IH, Chou CC, Chen CC. Protection from ultraviolet irradiation by melanin of mosquitocidal activity of Bacillus thuringiensis var. israelensis. J Invertebr Pathol. 1993;62(2):131-6.

3. Patel KR, Wyman JA, Patel KA, Burden BJ. A Mutant ofBacillus thuringiensisProducing a Dark-Brown Pigment with Increased UV Resistance and Insecticidal Activity. J Invertebrate Pathol. 1996;67(2):120-4

4. Saxena D, Ben-Dov E, Manasherob R, Barak Z, Boussiba S, Zaritsky A. A UV tolerant mutant of Bacillus thuringiensis subsp. kurstaki producing melanin. Curr Microbiol. 2002;44(1):25-30.

5. Chen G, Wang GY, Li X, Waters B, Davies J. Enhanced production of microbial metabolites in the presence of dimethyl sulfoxide. $J$ Antibiot (Tokyo). 2000;53(10):1145-53.

6. Hoti SL, Balaraman K. Formation of melanin pigment by a mutant of Bacillus thuringiensis H-14. J General Microbiol. 1993;139(10):2365-9.

7. Vilas-Bôas GT, Vilas-Bôas LA, Braz VT, Saridakis HO, Santos CA, Arantes OMN. Isolation and partial characterization of a mutant of Bacillus thuringiensis producing melanin. Brazilian J Micro- biol. 2005;36(3):271-4.

8. Ruan L, Yu Z, Fang B, He W, Wang Y, Shen P. Melanin pigment formation and increased UV resistance in Bacillus thuringiensis following high temperature induction. Syst Appl Microbiol. 2004;27(3):286-9.

9. Liu F, Yang W, Ruan L, Sun M. A Bacillus thuringiensis host strain with high melanin production for preparation of light-stable biopesticides. Annals Microbiol. 2012;63(3):1131-5.

10. Arnow LE. Colorimetric determination of the components of 3, 4-dihydroxyphenylalaninetyrosine mixtures. J Bio Chem. 1937;118(2):531-7.

11. Dulmage HT, Correa JA, Martinez AJ. Coprecipitation with lactose as a means of recovering the spore-crystal complex of Bacillus thuringiensis. J Invertebrate Pathol.1970;15(1):15-20.

12. Laemmli UK. Cleavage of structural proteins during the assembly of the head of bacteriophage T4. Nature.1970;227(5259):680-5.

13. Rojas-Ruiz NE, Vazquez-Cruz C, Sanchez-Alonso P, SansineneaRoyano E. POPULATION ANALYSIS OF Bacillus thuringiensis IN INTERACTION in vitro WITH SOIL BACTERIA. Agrociencia. 2010;44(8):941-53.

14. Pozsgay M, Fast P, Kaplan H, Carey PR. The effect of sunlight on the protein crystals from Bacillus thuringiensis var. kurstaki HD and NRD12: A Raman spectroscopic study. J Invertebrate Pathol. 1987;50(3):246-53.

15. Pusztai M, Fast P, Gringorten L, Kaplan H, Lessard T, Carey PR The mechanism of sunlight-mediated inactivation of Bacillus thuringiensis crystals. Biochem J.1991;273(Pt 1):43-7.

16. Cohen E, Rozen H, Joseph T, Braun S, Margulies L. Photoprotection of Bacillus thuringiensis kurstaki from ultraviolet irradiation. J Invertebrate Pathol. 1991;57(3):343-51.

17. Dunkle RL, Shasha BS. Response of Starch-Encapsulated Bacillus thuringiensis Containing Ultraviolet Screens to Sunlight. Envir Entomol.1989;18(6):1035-41.

18. Sansinenea E, Ortiz A. Secondary metabolites of soil Bacillus spp. Biotechnol Lett. 2011;33(8):1523-38. 